\title{
Comparative Results of "Epi-Off" Conventional versus "Epi-Off” Accelerated Cross-Linking Procedure at 5-year Follow-Up
}

\author{
Cristina Ariadna Nicula $\mathbb{D}^{1,2}$ Dorin Nicula ${ }^{10}{ }^{2}$ Anca Maria Rednik (iD, ${ }^{3}$ \\ and Adriana Elena Bulboacă ${ }^{4}{ }^{4}$ \\ ${ }^{1}$ Department of Ophthalmology, Iuliu Haţieganu University of Medicine and Pharmacy, Cluj-Napoca, Romania \\ ${ }^{2}$ Oculens Clinic, Cluj-Napoca, Romania \\ ${ }^{3}$ County Eye Hospital, Cluj-Napoca, Romania \\ ${ }^{4}$ Department of Physiopathology, Iuliu Haţieganu University of Medicine and Pharmacy, Cluj-Napoca, Romania
}

Correspondence should be addressed to Cristina Ariadna Nicula; niculacristina65@yahoo.com

Received 27 May 2020; Accepted 22 June 2020; Published 23 July 2020

Academic Editor: Alessandro Meduri

Copyright (c) 2020 Cristina Ariadna Nicula et al. This is an open access article distributed under the Creative Commons Attribution License, which permits unrestricted use, distribution, and reproduction in any medium, provided the original work is properly cited.

\begin{abstract}
Purpose. The aim of our study was to compare the long-term efficacy and safety of "epi-off" conventional and "epi-off" accelerated corneal cross-linking (CXL) in patients with progressive keratoconus. Methods. "Epithelial-off" ("Epi-off") CXL using the conventional technique $\left(3 \mathrm{~mW} / \mathrm{cm}^{2}, 30\right.$ minutes) was performed in 93 eyes of 93 patients (S-CXL group) and "epi-off" accelerated method $\left(9 \mathrm{~mW} / \mathrm{cm}^{2}, 10\right.$ minutes) in 76 eyes of 76 patients with progressive KCN (A-CXL group). Cases with different stages of keratoconus and topographic evidence of progression were included. Main outcomes comprised refraction, keratometry measurements, uncorrected (UCVA) and best-corrected visual acuity (BCVA), and topographical indices. Micromorphological analysis was assessed by anterior segment ocular coherence tomography (AS-OCT). The follow-up period was 5 years. Results. In both groups, Kflat presented similar results: decrease at 1 year $(p=0.465)$, at 2 years $(p=0.672)$, at 3 years $(p=0.198)$, at 4 years $(p=0.32)$, and at 5 years $(p=0.864)$. In both groups, Ksteep presented a similar decrease at 1 year $(p=0.709)$, at 2 years $(p=0.455)$, at 3 years $(p=0.43)$, at 4 years $(p=0.57)$, and at 5 years $(p=0.494)$, with no statistically significant difference. Decrease in Kavg was similar in both groups at all analyzed time points ( $p=0.18$ at 1 year, $p=0.093$ at 2 years, $p=0.57$ at 3 years, $p=0.154$ at 4 years, and $p=0.247$ at 5 years). Kmax had a similar decrease in both groups at 1 year $(p=0.06)$, at 2 years $(p=0.09)$, at 3 years $(p=0.126)$, at 4 years $(p=0.113)$, and at 5 years $(p=0.114)$. There was no statistically significant difference between the cylinder decrease in both groups ( $p=0.349$ at 1 year, $p=0.6782$ at 2 years, $p=0.299$ at 3 years, $p=0.0943$ at 4 years, and $p=0.144$ at 5 years). The BCVA values were statistically significantly higher than the preoperative values in both groups at all time points $(p<0.05)$. Topographical indices such as thinnest corneal point (TP), corneal volume (CV), index vertical asymmetry (IVA), index of vertical asymmetry (ISV), index of height asymmetry (IHA), index of height decentration (IHD), Belin/Ambrosio Enhanced Ectasia Display (BAD_D), and Ambrosio retinal thickness (ART Max) were significantly statistically decreased compared with baseline at all time points, in both groups. Conclusion. "Epi-off" accelerated and conventional CXL have the same efficacy in terms of improvement in visual and topographic outcomes.
\end{abstract}

\section{Introduction}

Keratoconus $(\mathrm{KCN})$ is a bilateral ectatic corneal disorder, frequently asymmetric, with a progressive thinning of the cornea resulting in protrusion, progressive irregular astigmatism, and visual deterioration [1]. In the majority of cases, this condition affects young patients with an early age of onset as a negative prognostic factor for evolution and corneal transplantation [2]. That is why, an early detection of KCN by using the topographical and tomographical evaluation of the Pentacam device is of major importance $[3,4]$. During the past years, corneal collagen cross-linking (CXL) became the standard procedure for KCN therapy. CXL has been used in dentistry, orthopedics, and dermatology for 
many years, and since 1998, it has been tested and subsequently introduced in the treatment of KCN together with riboflavin (a nontoxic photosensitizing agent) and ultraviolet irradiation (UVA) [5]. The final effect of the CXL technique is represented by the strengthening of the cornea, and the goal is to slow down or stop the progression of $\mathrm{KCN}$, thus avoiding or delaying the necessity of keratoplasty.

CXL technique consists in the photopolymerization of the stromal fibrillar tissue, in order to increase their stiffness and resistance to corneal ectasia and proteolytic enzymes (collagenase) [6,7], reducing corneal permeability [8] and formation of large collagen molecular aggregates [9] through the combined action of the photosensitizing substance (riboflavin-B2) and ultraviolet A (UVA) light irradiation performed with an illuminator in a solid state of UVA kind $[5,9]$. The conventional (standard) technique ("epi-off" technique) named as the Dresden protocol uses riboflavin which is exposed to a measured dose of long wavelength UVA radiation $(370 \mathrm{~nm})$ at $3 \mathrm{mw} / \mathrm{cm}^{2}$ for 30 minutes, applied after epithelial removal and resulting in a total energy dose of 3.47 or a radiant exposure of $5.47 \mathrm{~cm}[2,9,10]$. Several studies demonstrated the safety and long-term efficacy of the conventional "epi-off" cross-linking in stabilizing progressive KCN [11-19].

In order to reduce the time of treatment and the patient discomfort and to avoid the excessive corneal dehydration and thinning that can occur for a period of 30 minutes, the accelerated "epi-off" CXL was introduced. By applying a higher intensity $\left(9 \mathrm{mw} / \mathrm{cm}^{2}\right)$ for a shorter period of time (10 minutes), the same level of radiant exposure as in the conventional CXL can be achieved [20,21]. This is according to Bunsen-Roscoe's law of reciprocity that showed that an increase of UVA irradiation associated with a reduced exposure time theoretically delivers a total energy dose to the tissue similar to that in the conventional treatment, with the same biological effect $[20,21]$. Previous ex vivo experiments on porcine corneas performed with high energy and short irradiation time settings have revealed similar results on the biomechanical properties compared to the standard protocol [20]. The potential advantages of the accelerated CXL technique include decreased exposure time, improved comfort of the patient, and inferior infection risk [22]. Furthermore, there are studies that demonstrated the efficiency of "epi-off" accelerated CXL [20, 23-25]. However, Hashemi et al. [26] compared the two-year changes in dynamic corneal response evaluated by Corvis ST, between $18 \mathrm{~mW} / \mathrm{cm}^{2}$ (5 minutes) and $9 \mathrm{~mW} / \mathrm{cm}^{2}(10$ minutes) CXL protocols. The conclusion was that both procedures increased corneal stiffness, especially the $9 \mathrm{~mW} / \mathrm{cm}^{2}$ procedure. Moreover, there are studies that compared the outcomes of "epi-off" conventional and accelerated CXL procedures and showed comparable effect in preserving keratometry parameters in both techniques [22, 27-33]. Furthermore, Rechichi et al. [34], in a prospective, multicenter, and interventional study, evaluated intraoperative corneal pachymetry in patients undergoing pulsed-light accelerated CXL with dextran-free hydroxyl-propyl methylcellulose $0.1 \%$ riboflavin solution (8 minutes of exposure, $30 \mathrm{~mW} / \mathrm{cm}^{2}$, and an energy dose of $7.2 \mathrm{~J} / \mathrm{cm}^{2}$ ) and revealed no statistically significant intraoperative corneal thickness reduction.

Evaluation of the demarcation line is considered as a measurement of the depth of CXL treatment into the stroma [35]. Corneas that underwent CXL treatment present an inferior rate of enzymatic collagenase degradation when compared to an untreated cornea [35]. Some studies $[36,37,39]$ showed the role of the demarcation line after CXL depth as representative of CXL effectiveness.

The aim of our study was to assess the long-term efficacy and safety of "epi-off" conventional and accelerated CXL by comparing the keratometry measurements, spherical and cylinder equivalents, visual acuity, and topographical indices in patients with progressive keratoconus up to 5 years, following the procedure, and also to determine the qualitative corneal changes evaluated by anterior segment optical coherence tomography (AS-OCT) after both procedures.

\section{Materials and Methods}

A retrospective single-center study was performed at the Oculens Private Clinic in Cluj-Napoca, Romania, after the unanimous approval of the clinic ethics committee (5/2020) and adhered to the tenets of Helsinki Declaration (1964). Two groups of patients were included in our study. The conventional group included 93 eyes of 93 patients, with progressive $\mathrm{KCN}$ who underwent CXL by the conventional (standard) "epi-off" technique (S-CXL group). The accelerated group included 76 eyes of 76 patients, with progressive KCN who underwent CXL by the accelerated "epi-off" procedure (A-CXL group). All patients included in the study were affected by KCN with a documented clinical and instrumental worsening at least in the last three to six months of observation: myopia and astigmatism changes $>3 \mathrm{D}$, a mean change of central $\mathrm{K}$ value $>1.5 \mathrm{D}$ in three consecutive corneal topographic measurements, increase in the maximum keratometry (Kmax) in topography of more than 1D, or a mean decrease in central corneal thickness $>5 \%$ in three consecutive tomographic measurements [39]. The "epi-off" conventional CXL was performed between January 2011 and January 2013 and the "epi-off" accelerated CXL between February 2013 and January 2015. All cases were followed up postoperatively at 1 month, 6 months, and $1,2,3,4$, and 5 years, following the procedure.

The inclusion criteria for both groups included age greater than 18 years, any gender, progressive KCN of different stages (according to the Amsler-Krumeich classification), and an average corneal thickness of at least $400 \mu \mathrm{m}$ at the thinnest corneal location. Patients with previous intracorneal ring placement, corneal pachymetry less than $400 \mu \mathrm{m}$, Vogt striae presence, history of herpetic keratitis or other corneal infections, dry eye syndrome, aphakia, central corneal scar, autoimmune illnesses, nystagmus, and pregnancy were excluded.

Before the procedure, a complete ocular assessment was performed, including uncorrected (UCVA) and best-corrected visual acuity (BCVA), refractometry (manifest and cycloplegic), keratometry-steep (Ksteep), flat (Kflat), average (Kavg), and maximum (Kmax) (Topcon auto refracto- 
keratometer, KR 8900, Japan), slit-lamp exam (Slit Lamp BX 900, Haag-Streit AG3098 Koeniz, Switzerland), eye fundus examination, intraocular pressure measured by applanation tonometry, corneal tomography (Pentacam ${ }^{\circledR}$ HR Premium; Oculus Optikgerate GmbH, Wetzlar, Germany), and endothelial cell counting (Konan SP-9000, Hyogo, Japan). Visual acuity was measured with the Snellen charts. For scientific reasons, it was transformed as the logarithm of minimum angle of resolution (LogMar). Patients were requested to discontinue the wear of contact lens 2 weeks before evaluation or surgery.

After signing the specific informed consent, the two groups of patients underwent the "epi-off" conventional or "epi-off" accelerated CXL procedure.

The CXL procedure was performed in the operating room in sterile conditions. The "epi-off" CXL technique was used for both conventional and accelerated procedures. During the procedure, a single $3.0 \mathrm{ml}$ of riboflavin $0.1 \%$ dextran 20\% solution (Peschke D) was opened, and the power of the UVA illuminator (Peschke Meditrade GmbH, Huenenberg, Switzerland CXL system) was verified. Before the procedure, one drop of Isopto ${ }^{\circledR}$ Carpine (Amman Pharma, Romania) was instilled in the eye and was prepared with topical anesthesia with oxybuprocaine hydrochloride 0.4\% (Benoxi, Unimed Pharma Ltd), 1-2 drops, 10-15 minutes prior to cross-linking was instilled. Sterile operating field and lid speculum were used. A corneal de-epithelization with a blunt spatula on a $9 \mathrm{~mm}$ diameter optical zone was performed, followed by the instillation of riboflavin $0.1 \%$ dextran 20\% solution (Peschke ${ }^{\circledR}$ D, Huenenberg, Switzerland), every 2 minutes for 30 minutes before irradiation. Pachymetry was done after epithelial removal to ascertain that the corneal depth was more than $400 \mathrm{~m}$. The optical corneal zone was additionally aligned under a UVA power of $3 \mathrm{~mW} / \mathrm{cm}^{2}$ for 30 minutes in conventional CXL (Dresden protocol) (total energy:7.2 J/cm ${ }^{2}$ ) and under a power of $9 \mathrm{~mW} / \mathrm{cm}^{2}$ for 10 minutes in accelerated CXL (total energy: $5,4 \mathrm{~J} / \mathrm{cm}^{2}$ ). Riboflavin $0.1 \%$ every 2 minutes was instilled during irradiation in both groups. The cornea surface was irrigated with balanced salt solution after irradiation. At the end of the surgery, steroids and antibiotics (Tobradex) (Alcon Novartis, Dallas, Worth, USA) were instilled. A bandage soft contact lens was applied for 3 days until the corneal healing was completed. After the procedure, all patients received topical antibiotics and steroids such as Tobradex (Alcon Novartis, Dallas, Worth, USA) 5 times/day, for one month, and artificial tears 2 times/day for 2 to 3 months. Both groups were followed up postoperatively in the clinic at day 1 , day 3 , month 1 , month 6 , year 1 , and yearly up to 5 years. Ocular refraction, keratometry measurements, uncorrected visual acuity (UCVA), best-corrected visual acuity (BCVA), slit-lamp examination, corneal tomography, and topography were performed at all visits, except for the first postoperative day, 4 weeks, and 6 months. Corneal topographic and aberrometer parameters were recorded from all the examinations. At one month after the procedure (in both groups), anterior segment optical coherence tomography (AS-OCT) analysis (Triton OCT, Topcon Corporation, Tokyo, Japan) was done in order to assess qualitative A-CXL- and S-CXL-induced corneal changes and treatment penetration looking after demarcation line. The image was captured when the corneal reflex was visible, and the depth of demarcation line was measured using the caliper tool provided by the manufacturer.

2.1. Statistical Analysis. Data were presented using the number (percentage) for categorical variables and mean\pm standard deviation or median (quartile 1; quartile 3) for continuous variables. Groups were compared using the independent Student $t$-test for continuous variables with normal distribution, the median test for continuous variables with abnormal distribution, the and chi-square test for categorical variables. The evolution of variables at different time points was compared with baseline values using the paired $t$-test. A $p$ value $<0.05$ was considered as statistically significant.

\section{Results}

We analyzed data of 93 eyes of 93 patients with progressive $\mathrm{KCN}$ who were treated with the conventional (standard) "epi-off" CXL technique (S-CXL group) and 76 eyes of 76 patients treated with the "epi-off" accelerated CXL (A-CXL group). At baseline, the mean age was $26.5 \pm 8.5$ years (ranging between 18 and 54 years) in the S-CXL group and $29 \pm 8.2$ years (ranging between 18 and 48 years) in the A-CXL group ( $p$ value for the difference between group$s=0.064)$. There were 52 males $(55.9 \%)$ in the S-CXL group and 41 males $(53.9 .3 \%)$ in the A-CXL group $(p=0.184)$. Regarding the stage of keratoconus (Amsler-Krumeich staging), $8.6 \%$ had stage I, $53.76 \%$ of patients had stage II, and $37.63 \%$ stage III in the S-CXL group. In the A-CXL group, $7.89 \%$ had stage I, $47.37 \%$ of patients had stage II, and $44.74 \%$ stage III (Table 1 ).

3.1. Corneal Findings during the Treatment. There was no statistically significant difference between study groups in terms of preoperative flat keratometry (Kflat) $(p=0.293)$, steep keratometry (Ksteep) $(p=0.098)$, mean keratometry (Kavg) $(p=0.309)$, and $\operatorname{Kmax}(p=0.179)$. In the S-CXL group, Kflat dropped from $47.47 \pm 4.15 \mathrm{D}$ to $46.45 \pm 3.94 \mathrm{D}$ at 1 year $(p=0.037)$, to $46.4 \pm 4.00 \mathrm{D}(p=0.025)$ at 2 years, to $46.39 \pm 3.99 \mathrm{D}(p=0.0098)$ at 3 years, to $46.37 \pm 3.71 \mathrm{D}$ at 4 years $(p=0.0046)$, and to $46.29 \pm 3.69 \mathrm{D}(p=0.0064)$ at 5 years. In the A-CXL group, Kflat dropped from $46.67 \pm 4.34 \mathrm{D}$ to $46.15 \pm 4.45 \mathrm{D}$ at 1 year $(p=0.0433)$, to $46.1 \pm 4.40 \mathrm{D}$ at 2 years $(p=0.0049)$, to $45.98 \pm 4.36 \mathrm{D}$ at 3 years $(p=0.039)$, to $45.93 \pm 4.38 \mathrm{D}$ at 4 years $(p=0.0066)$, and to $45.88 \pm 4.39 \mathrm{D}(p=0.0039)$ at 5 years. Kflat presented a similar decrease in both groups at 1 year $(p=0.465)$, at 2 years $(p=0.672)$, at 3 years $(p=0.198)$, at 4 years $(p=0.32)$, and at 5 years $(p=0.864)$ (Table 2 and Figure 1$)$.

In the S-CXL group, Ksteep dropped from $50.42 \pm 4.87 \mathrm{D}$ to $49.54 \pm 4.71 \mathrm{D}$ at 1 year $(p=0.0043)$, to $49.45 \pm 4.81 \mathrm{D}$ $(p=0.0023)$ at 2 years, to $49.43 \pm 4.81 \mathrm{D}(p<0.0001)$ at 3 years, to $49.42 \pm 4.90 \mathrm{D}$ at 4 years $(p=0.0012)$, and to $49.40 \pm 4.88 \mathrm{D}(p=0.0008)$ at 5 years. In the A-CXL group, 
TABLE 1: Gender, age, and keratoconus stage in both groups.

\begin{tabular}{|c|c|c|c|}
\hline Parameter & $\begin{array}{c}\text { Conventional CXL (S-CXL) } \\
N=93 \text { eyes ( } 93 \text { patients) }\end{array}$ & $\begin{array}{l}\text { Accelerated CXL (A-CXL) } \\
N=76 \text { eyes ( } 76 \text { patients) }\end{array}$ & $p$ value \\
\hline Men, $n(\%)$ & $52(55.9)$ & $41(53.9)$ & 0.184 \\
\hline Mean age, years & $26.5 \pm 8.5$ & $29 \pm 8.2$ & 0.064 \\
\hline \multicolumn{4}{|c|}{ Keratoconus stage, $n(\%)$} \\
\hline I & $8(8.6)$ & $6(7.89)$ & $<0.001$ \\
\hline II & $50(53.76)$ & $36(47.37)$ & \\
\hline III & $35(37.63)$ & $34(44.74)$ & \\
\hline
\end{tabular}

Ksteep dropped from $49.98 \pm 4.51 \mathrm{D}$ to $49.22 \pm 4.11 \mathrm{D}$ at 1 year $(p=0.0072)$, to $49.08 \pm 4.61 \mathrm{D}$ at 2 years $(p=0.0009)$, to $48.99 \pm 4.56 \mathrm{D}$ at 3 years $(p=0.027)$, to $48.91 \pm 4.54 \mathrm{D}$ at 4 years $(p=0.0036)$, and to $48.88 \pm 4.54 \mathrm{D}$ at 5 years $(p=0.002)$ (Table 2 and Figure 1). Ksteep presented a similar decrease in both groups at 1 year $(p=0.709)$, at 2 years $(p=0.455)$, at 3 years $(p=0.43)$, at 4 years $(p=0.57)$, and at 5 years $(p=0.494)$, with no statistically significant difference (Table 2 and Figure 2).

For Kavg, the decrease was similar in both groups at all time points analyzed. In the S-CXL group, Kavg dropped from $48.95 \pm 4.16 \mathrm{D}$ to $48.00 \pm 4.06 \mathrm{D}(p=0.0194)$ at 1 year, to $47.93 \pm 4.10 \mathrm{D}(p=0.0067)$ at 2 years, to $47.91 \pm 4.10 \mathrm{D}$ $(p=0.0074)$ at 3 years, to $47.90 \pm 4.30 \mathrm{D}(p=0.0211)$ at 4 years, and at to $47.89 \pm 4.30$ at 5 years $(p=0.011)$. In the A-CXL group, Ksteep dropped from $48.33 \pm 4.14 \mathrm{D}$ to $47.69 \pm 4.36 \mathrm{D}$ at 1 year $(p=0.01)$, to $47.59 \pm 4.32 \mathrm{D}$ $(p=0.022)$ at 2 years, to $47.49 \pm 4.33 \mathrm{D}(p=0.0068)$ at 3 years, to $47.42 \pm 4.30 \mathrm{D}(p=0.008)$ at 4 years, and to $47.40 \pm 4.30$ at 5 years $(p=0.0053)$ (Table 2 and Figure 3 ). The reduction of Kavg from baseline data was $1.06 \mathrm{D}$ in the S-CXL group and $0.93 \mathrm{D}$ in the A-CXL group.

For Kmax, the decrease was similar in both groups at all time points analyzed. In the S-CXL group, Kmax dropped from $54.05 \pm 5.41 \mathrm{D}$ to $52.10 \pm 5.35(p=0.0172)$ at 1 year, to $51.99 \pm 5.35 \mathrm{D}(p=0.012)$ at 2 years, to $51.78 \pm 5.28 \mathrm{D}$ $(p=0.0086)$ at 3 years, to $51.74 \pm 5.31 \mathrm{D}(p=0.0075)$ at 4 years, and at to $51.72 \pm 5.32$ at 5 years $(p=0.0071)$. In the A-CXL group, Kmax dropped from $56.07 \pm 5.39 \mathrm{D}$ to $54.45 \pm 5.42 \mathrm{D}$ at 1 year $(p=0.0456)$, to $54.22 \pm 5.44 \mathrm{D}$ $(p=0.0368)$ at 2 years, to $54.02 \pm 5.38 \mathrm{D}(p=0.0199)$ at 3 years, to $54.01 \pm 5.38 \mathrm{D}(p=0.0196)$ at 4 years, and to $54.00 \pm 5.39 \mathrm{D}$ at 5 years $(p=0.0189)$ (Figure 4$)$. There was a statistically significant decrease in $\mathrm{Kmax}$ in both groups comparing with baseline at all time points (Table 2). The reduction of Kmax from baseline data was 2.33 in the S-CXL group and $2.07 \mathrm{D}$ in the A-CXL group.

Compared to preoperative status, for conventional and accelerated CXL, Kflat, Ksteep, Kavg, and Kmax were statistically significantly lower at year 1 and were maintained statistically significantly lower at all time points $(p<0.05$ for all time points as compared to preoperative values) (Table 2).

In the S-CXL group, mean cylinder decreased from $-4.415 \pm 2.39 \mathrm{D}$ to $-3.905 \pm 2.26$ at 1 year $(p=0.04)$, to $-3.435 \pm 2.22 \mathrm{D}$ at 2 years $(p=0.0093)$, to $-3.37 \pm 2.27 \mathrm{D}$ at 3 years $(p=0.0399)$, to $-3.361 \pm 2.26 \mathrm{D}$ at 4 years $(p=0.042)$, and to $-3.358 \pm 2.27 \mathrm{D}$ at 5 years $(p=0.033)$. In the A-CXL group, mean cylinder decreased from $-4.15 \pm 2.15 \mathrm{D}$ to
$-3.661 \pm 2.19 \mathrm{D}$ at 1 year $(p=0.033)$, to $-3.105 \pm 2.21 \mathrm{D}$ at 2 years $(p=0.028)$, to $-3.076 \pm 2.17 \mathrm{D}$ at 3 years $(p=0.0062)$, to $-3.002 \pm 2.18 \mathrm{D}$ at 4 years $(p=0.0309)$, and to $-2.997 \pm 2.22$ at 5 years $(p=0.022)$. There was a statistically significant difference in the cylinder value between baseline and all time point visits. There was no statistically significant difference between the decrease in the cylinder value between the two groups $(p=0.349$ at 1 year; $p=0.6782$ at 2 years; $p=0.299$ at 3 years; $p=0.0943$ at 4 years; $p=0.081$ at 5 years) (Figure 5 ).

Compared to baseline, in the S-CXL group, spherical equivalent (SE) decreased from $-6.1 \pm 4.2 \mathrm{D}$ to $-5.48 \pm 3.93 \mathrm{D}$ at 1 year $(p=0.0065)$, to $-5.1 \pm 4.01 \mathrm{D}$ at 2 years $(p=0.0005)$, to $-5 \pm 4.12 \mathrm{D}$ at 3 years $(p=0.0128)$, to $-4.92 \pm 3.87 \mathrm{D}$ at 4 years $(p=0.0179)$, and to $-4.9 \pm 3.88 \mathrm{D}$ at 5 years $(p=0.0166)$. In the A-CXL group, SE decreased from baseline $-5.89 \pm 4 \mathrm{D}$ to $-5.27 \pm 4.07 \mathrm{D}$ at 1 year $(p=0.0007)$, to $-5.02 \pm 3.97 \mathrm{D}$ at 2 years $(p=0.0081)$, to $-4.87 \pm 3.79 \mathrm{D}$ at 3 years $(p=0.004)$, to $-4.82 \pm 4.1 \mathrm{D}$ at 4 years $(p=0.01)$, and to $-4.79 \pm 4.1 \mathrm{D}$ at 5 years $(p=0.0109)$, with no statistically significant difference between groups at any time point $(p=0.2119$ at 1 year; $p=0.92$ at 2 years; $p=0.4803$ at 3 years; $p=0.1866$ at 4 years; $p=0.087$ at 5 years) (Figure 6).

There was no statistically significant difference in UCVA and BCVA between conventional and accelerated CXL in comparison with baseline values $(p=0.6283 / p=0.543)$. The preoperative UCVA in the S-CXL group was $0.75 \pm 0.2 \mathrm{LogMar}$ and remained $0.7 \pm 0.22 \mathrm{LogMar}$ at 1 year. At 2 years, UCVA increased to $0.68 \pm 0.22 \mathrm{LogMar}$, to $0.67 \pm 0.18 \mathrm{LogMar}$ at 3 years, to $0.67 \pm 0.21$ LogMar at 4 years, and to $0.67 \pm 0.20$ at 5 years. The preoperative UCVA in the A-CXL group was $0.73 \pm 0.19$ LogMar and increased to $0.69 \pm 0.24 \operatorname{LogMar}$ at 1 year, to $0.67 \pm 0.23 \mathrm{LogMar}$ at 2 years, to $0.65 \pm 0.21 \mathrm{LogMar}$ at 3 years, to $0.65 \pm 0.24 \operatorname{LogMar}$ at 4 years, and became $0.65 \pm 0.23$ LogMar at 5 years. For BCVA, the improvement was not statistically significantly different between the A-CXL group and the S-CXL group at 1 year $(p=0.1142)$, at 2 years $(p=0.908)$, at 3 years $(p=0.346)$, at 4 years $(p=0.4575)$, and at 5 years $(p=0.4072)$. Compared to baseline, in the S-CXL group, postoperative UCVA was statistically significantly lower than preoperative values at all time points analyzed $(p<0.001$ for all time points). In the A-CXL group, postoperative UCVA was statistically significantly higher compared to baseline starting from year 1 and maintained higher than preoperative levels at years 3, 4, and $5(p<0.001)$ (Figure 7).

For BCVA, the values were statistically significantly higher than the baseline values in both groups at all time points $(p<0.05)$ (Figure 8$)$. 


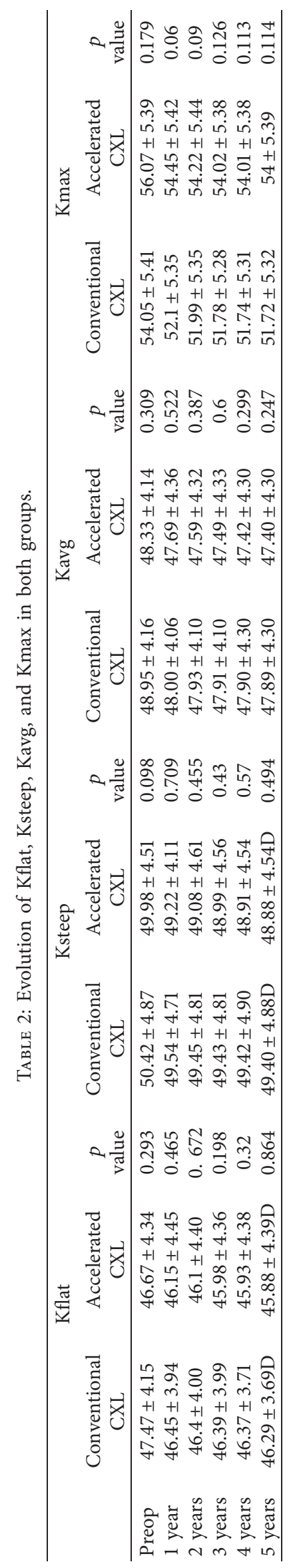




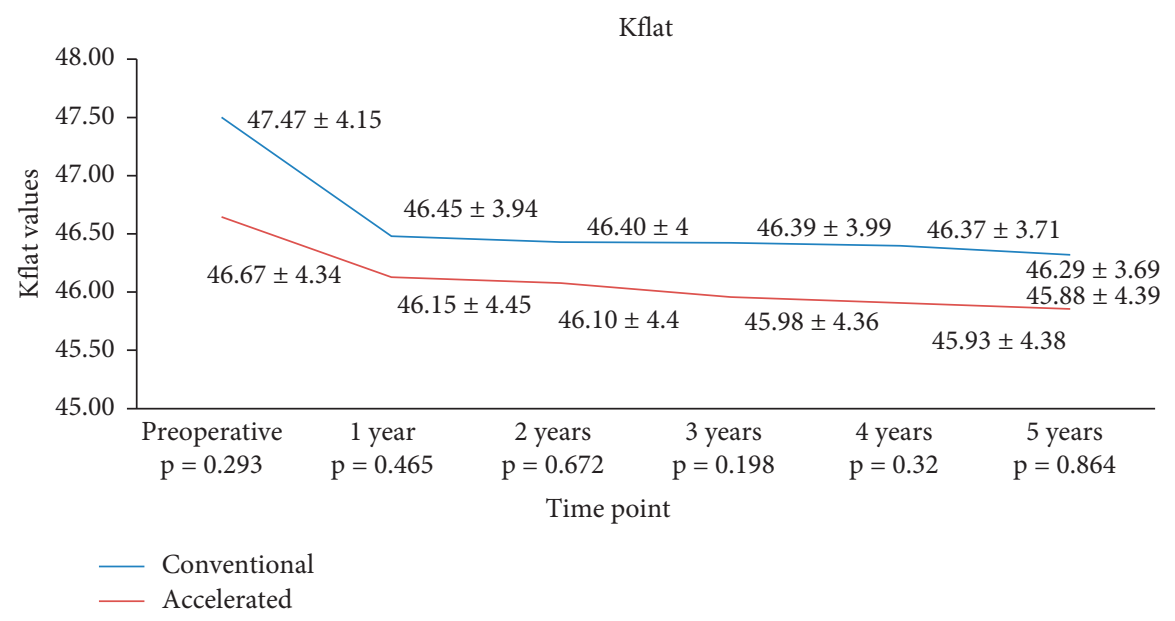

Figure 1: Evolution of Kflat in both groups.

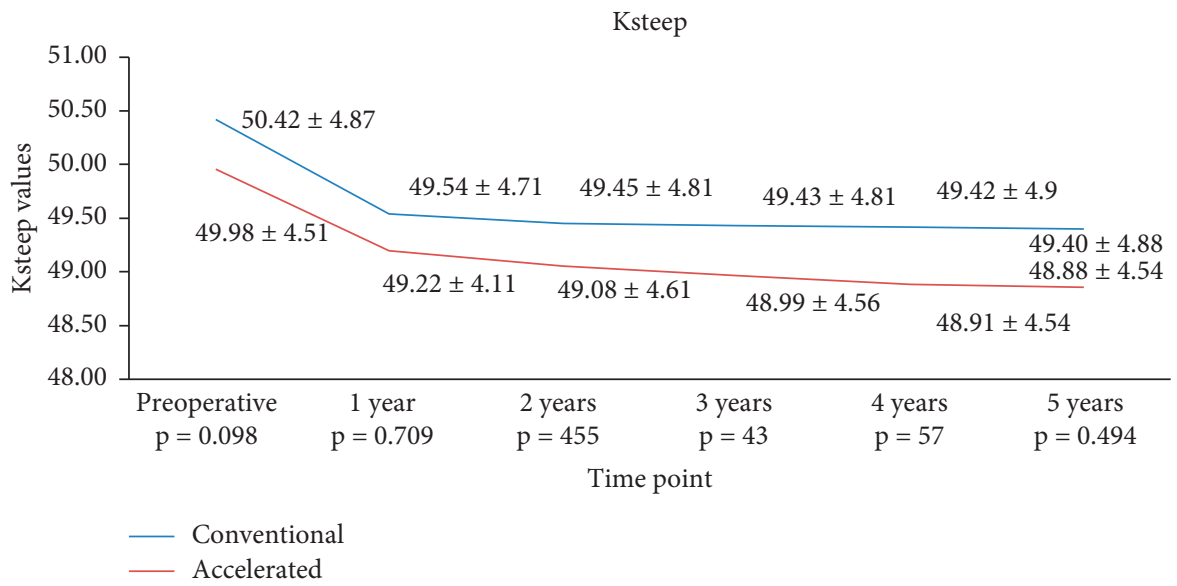

FIGURE 2: Evolution of Ksteep in both groups.

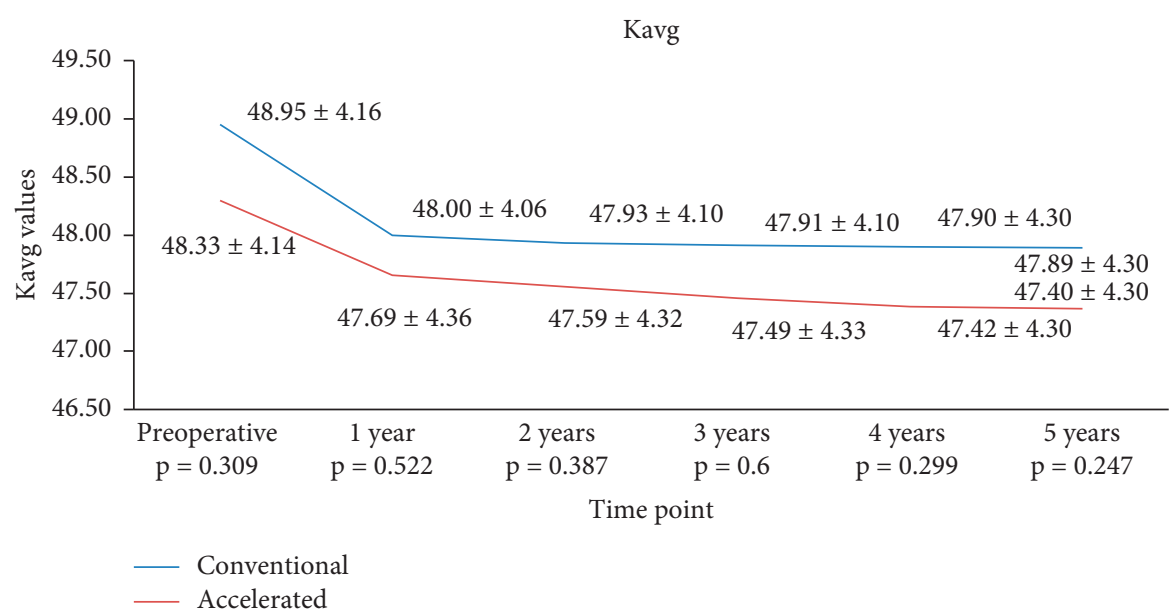

FIGURE 3: Evolution of Kavg in both groups.

3.2. Topographical Indices. Thinnest corneal point (TP), corneal volume (CV), index vertical asymmetry (IVA), index of vertical asymmetry (ISV), index of height asymmetry (IHA), index of height decentration (IHD), Belin/Ambrosio
Enhanced Ectasia Display (BAD_D), and Ambrosio retinal thickness (ART Max) were significantly statistically decreased compared with baseline at all time points in both groups (Table 3 ). We did not find any significantly 


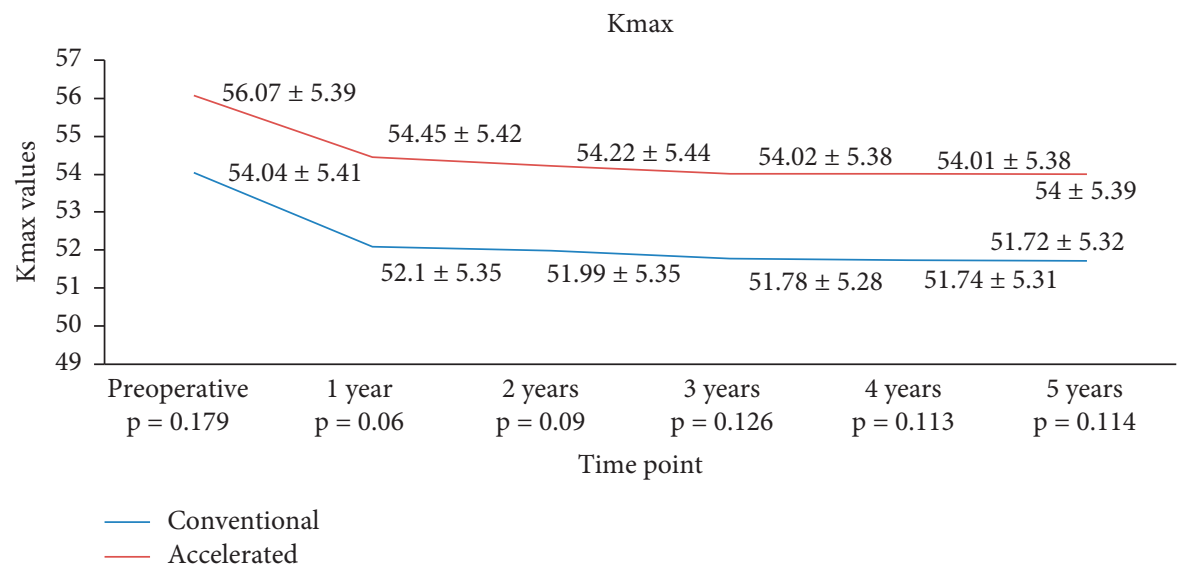

Figure 4: Evolution of Kmax in both groups.

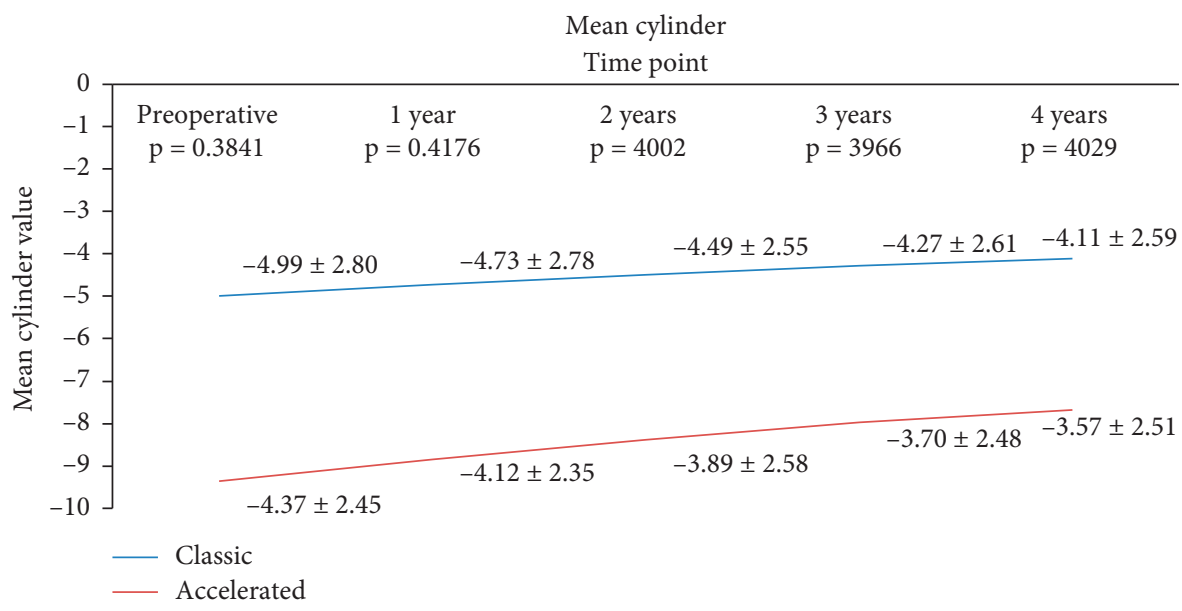

Figure 5: Evolution of cylinder in both groups.

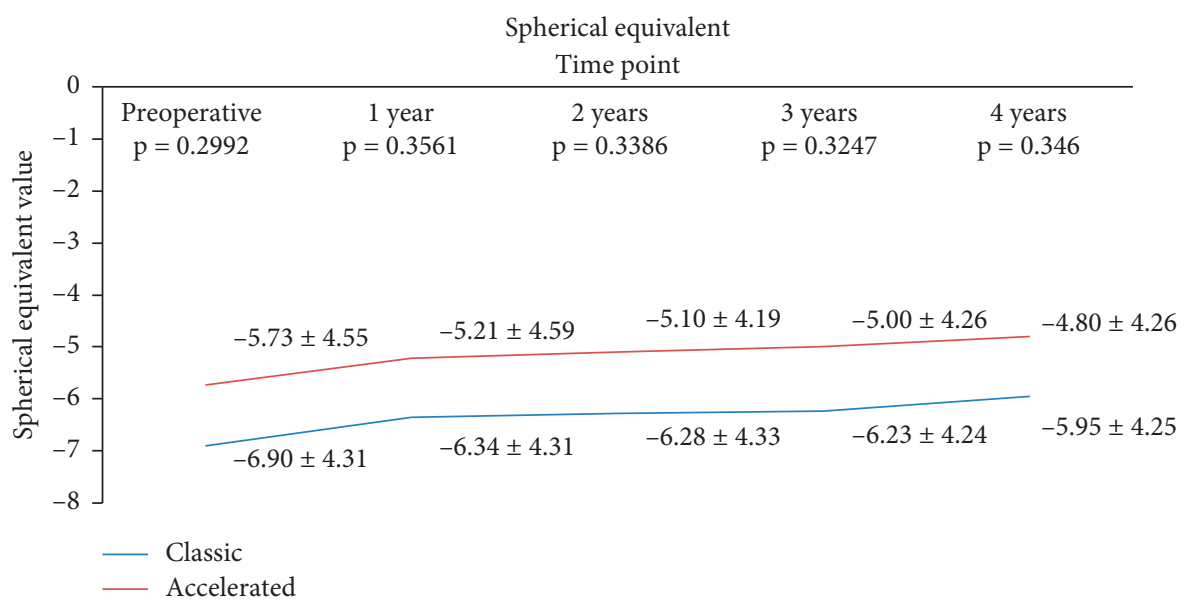

FIgURE 6: Evolution of SE in both groups.

statistically difference in the above parameters between S-CXL and A-CXL groups $(p>0.05)$ (Table 3$)$.

The changes in corneal total high ocular aberration (HOA) and root mean square values (RMS) compared with the baseline decreased significantly and statistically in both groups $(p<0.05$ and $p<0.005$, respectively) but did not differ significantly between $\mathrm{S}-\mathrm{CXL}$ and A-CXL groups (Table 4). 


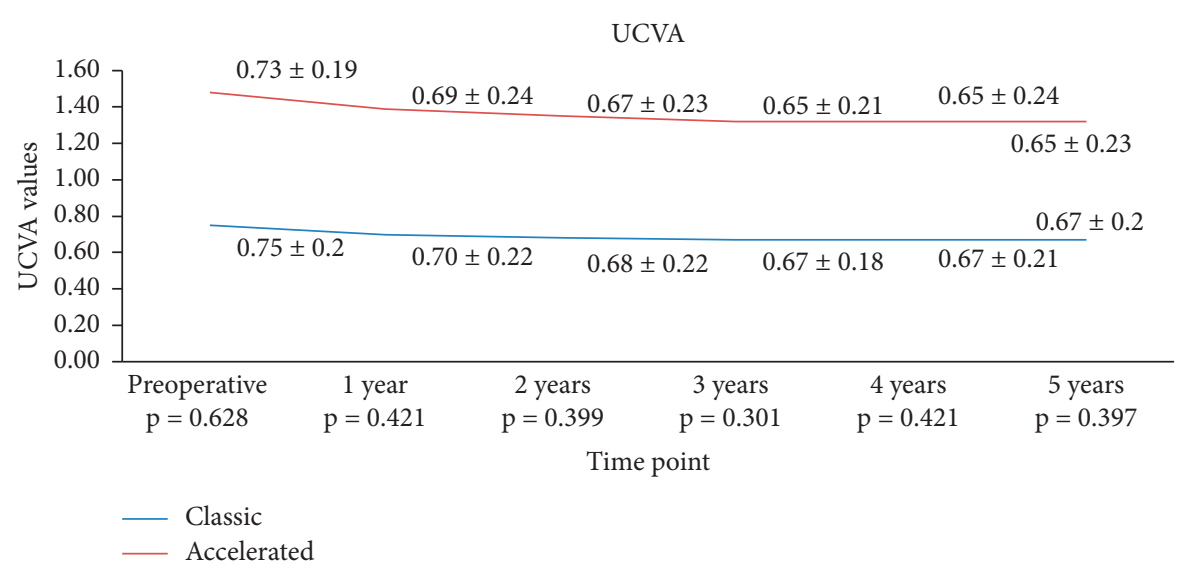

Figure 7: Evolution of UCVA in both groups.

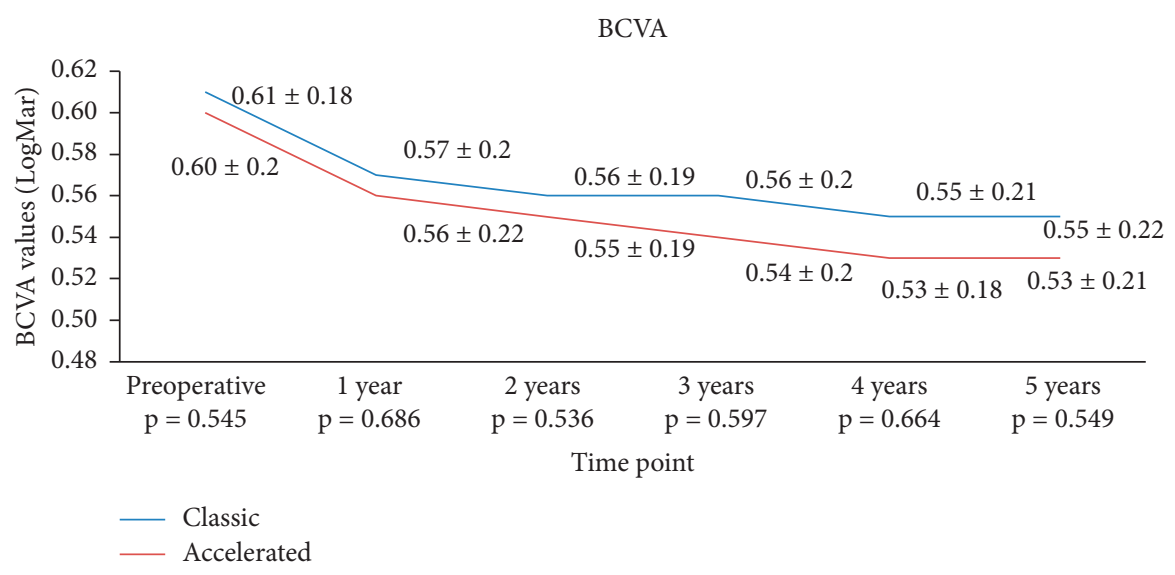

Figure 8: Evolution of BCVA in both groups.

There was no statistically significant difference in the corneal stromal demarcation line depth between the two groups, with a mean depth of $214 \pm 12.82 \mu \mathrm{m}$ in the S-CXL group and $203 \pm 12.03 \mu \mathrm{m}$ in the A-CXL group $(p=0.0736)$.

None of our patients in none of the groups lost lines of BCVA. In each case in both groups, complete epithelization was accomplished in 3 days. Infections or melting were not noticed in any of the studied group cases. Haze was present in the majority of cases in both groups for about 3 to 6 months but decreased progressively after this period.

\section{Discussion}

With recent modifications of the original Dresden protocol, accelerated CXL has become one of the interesting topics in corneal surgery. It has been shown that "epi-off" standard CXL (Dresden protocol) leads to stabilization of KC, with flattening of topographic keratometry and improvement of visual acuity in many cases $[19,40-44]$.

In our study, we aimed to evaluate the evolution of patients up to 5 years, following "epi-off" standard and accelerated CXL procedures. Results of our study showed a similar reduction in Kavg, Ksteep, and Kflat in both accelerated and conventional study groups at all time points $(p<0.05)$, maintained at 5 years. In a comparative study of accelerated $\left(30 \mathrm{~mW} / \mathrm{cm}^{2}\right.$ for 3 minutes at $5.4 \mathrm{~J} / \mathrm{cm}^{2}$ ) versus conventional CXL (Dresden protocol), Tomita et al. [30] reported for the first time a significant flattening of keratometry measurements in both groups at 1 year, following the procedure, with no statistically significant difference between the study groups $(\Delta K=-0.62 \mathrm{D}$ in the accelerated group and $\Delta \mathrm{K}=-1.77 \mathrm{D}$ in the conventional group, $p=0.21$ ). They also showed that the difference between the mean demarcation line depth in both groups was not statistically significant $(294.38 \pm 60.57 \mu \mathrm{m}$ in the accelerated group and $380.78 \pm 54.99 \mu \mathrm{m}$ in the conventional group). Furthermore, in a report which compared the results of accelerated $\left(7 \mathrm{~mW} / \mathrm{cm}^{2}\right.$ irradiation 15 minutes protocol $)$ versus conventional CXL (Dresden protocol), Kanellopoulos [45] reported the flattening of steep keratometry and the stabilization of $\mathrm{KCN}$ in both groups (from $49.5 \mathrm{D}$ to $46.1 \mathrm{D}$ in the accelerated group and from $48.7 \mathrm{D}$ to $45.8 \mathrm{D}$ in the conventional group). Similarly, Shetty et al. [46] revealed that the accelerated CXL $\left(9 \mathrm{~mW} / \mathrm{cm}^{2}\right.$ for 10 minutes and $18 \mathrm{~mW} / \mathrm{cm}^{2}$ for 5 minutes) had comparable outcomes to standard CXL, but the accelerated CXL using $30 \mathrm{~mW} / \mathrm{cm}^{2}$ for 3 minutes was not as efficient. In another study, in which 77 eyes treated with accelerated CXL and 76 treated with conventional CXL were enrolled and followed for 15 months, Hashemian et al. [47] showed a similar decrease in Ksteep at 15 months in both study groups $(\Delta$ Ksteep $=-1.98 \mathrm{D}$ in the conventional group and 
TABLE 3: Topographical parameter evolution in both groups.

\begin{tabular}{|c|c|c|c|c|c|c|}
\hline & arameter & S-CXL & $\begin{array}{l}p \text { value compared to } \\
\text { baseline }\end{array}$ & A-CXL & $\begin{array}{c}p \text { value compared to } \\
\text { baseline }\end{array}$ & $\begin{array}{c}p \text { value between } \\
\text { groups }\end{array}$ \\
\hline \multirow{6}{*}{ TP } & Preoperative & $462.419 \pm 34.36$ & & $463.565 \pm 35.53$ & & 0.832 \\
\hline & 1 year & $445.698 \pm 38.90$ & 0.0022 & $450.052 \pm 33.34$ & 0.0168 & 0.4416 \\
\hline & 2 years & $444.043 \pm 38.98$ & 0.0008 & $447.223 \pm 33.25$ & 0.0039 & 0.574 \\
\hline & 3 years & $442.677 \pm 38.9$ & 0.0003 & $441.527 \pm 41.7$ & 0.0006 & 0.8532 \\
\hline & 4 years & $441.419 \pm 38.97$ & 0.0001 & $441.263 \pm 42.02$ & 0.0006 & 0.9863 \\
\hline & 5 years & $441.15 \pm 38.9$ & 0.0001 & $441.132 \pm 42.23$ & 0.0006 & 0.9518 \\
\hline \multirow{6}{*}{ Vol C } & Preoperative & $57.452 \pm 6.55$ & & $57.069 \pm 3.46$ & & 0.6463 \\
\hline & 1 year & $56.173 \pm 6.55$ & 0.018 & $55.803 \pm 3.28$ & 0.0221 & 0.6547 \\
\hline & 2 years & $56.136 \pm 6.53$ & 0.0179 & $55.464 \pm 3.27$ & 0.0038 & 0.4151 \\
\hline & 3 years & $56.103 \pm 6.55$ & 0.0162 & $55.064 \pm 3.63$ & 0.0007 & 0.2182 \\
\hline & 4 years & $56.104 \pm 6.55$ & 0.0162 & $55.059 \pm 3.64$ & 0.0006 & 0.2179 \\
\hline & 5 years & $56.093 \pm 6.56$ & 0.0159 & $55.058 \pm 3.64$ & 0.0006 & 0.2236 \\
\hline \multirow{6}{*}{ IVA } & Preoperative & $0.900 \pm 0.41$ & & $1.001 \pm 0.32$ & & 0.0825 \\
\hline & 1 year & $0.760 \pm 0.4$ & 0.0199 & $0.851 \pm 0.32$ & 0.005 & 0.1149 \\
\hline & 2 years & $0.752 \pm 0.39$ & 0.0123 & $0.843 \pm 0.32$ & 0.0028 & 0.0851 \\
\hline & 3 years & $0.743 \pm 0.41$ & $<0.0001$ & $0.832 \pm 0.34$ & 0.0015 & 0.1131 \\
\hline & 4 years & $0.735 \pm 0.4$ & $<0.0001$ & $0.821 \pm 0.34$ & 0.0008 & 0.115 \\
\hline & 5 years & $0.730 \pm 0.41$ & $<0.0001$ & $0.815 \pm 0.32$ & 0.0004 & 0.217 \\
\hline \multirow{6}{*}{ ISV } & Preoperative & $79.354 \pm 29.53$ & & $89.578 \pm 27.53$ & & 0.222 \\
\hline & 1 year & $75.569 \pm 29.57$ & 0.0383 & $84.078 \pm 26.84$ & 0.0214 & 0.0541 \\
\hline & 2 years & $74.58 \pm 29.55$ & 0.0271 & $82.165 \pm 26.22$ & 0.0091 & 0.0892 \\
\hline & 3 years & $73.741 \pm 29.58$ & 0.0197 & $81.066 \pm 26.67$ & 0.0055 & 0.0964 \\
\hline & 4 years & $73.661 \pm 29.59$ & 0.0154 & $80.889 \pm 26.44$ & 0.0558 & 0.0731 \\
\hline & 5 years & $73.466 \pm 29.6$ & 0.0124 & $80.678 \pm 26.84$ & 0.0558 & 0.057 \\
\hline \multirow{6}{*}{ IHA } & Preoperative & $30.817 \pm 21.3$ & & $34.315 \pm 23.59$ & & 0.3141 \\
\hline & 1 year & $27.439 \pm 20.57$ & 0.0262 & $29.074 \pm 20.15$ & 0.0215 & 0.8265 \\
\hline & 2 years & $27.083 \pm 20.41$ & 0.0223 & $28.774 \pm 21.25$ & 0.0219 & 0.7823 \\
\hline & 3 years & $26.801 \pm 20.35$ & 0.019 & $28.362 \pm 20.63$ & 0.0103 & 0.6216 \\
\hline & 4 years & $26.553 \pm 20.3$ & 0.0164 & $28.023 \pm 21.02$ & 0.0101 & 0.6782 \\
\hline & 5 years & $26.315 \pm 20.28$ & 0.0141 & $27.974 \pm 21.22$ & 0.0101 & 0.7348 \\
\hline \multirow{6}{*}{ BAD_D } & Preoperative & $7.806 \pm 3.15$ & & $8.663 \pm 2.15$ & & 0.455 \\
\hline & 1 year & $7.593 \pm 3.14$ & 0.0445 & $8.220 \pm 2.22$ & 0.0397 & 0.1372 \\
\hline & 2 years & $7.548 \pm 3.15$ & 0.0057 & $8.170 \pm 2.08$ & 0.0352 & 0.1409 \\
\hline & 3 years & $7.51 \pm 3.15$ & 0.0152 & $8.150 \pm 2.19$ & 0.0164 & 0.13 \\
\hline & 4 years & $7.481 \pm 3.15$ & 0.0148 & $8.109 \pm 2.38$ & 0.0134 & 0.1136 \\
\hline & 5 years & $7.462 \pm 3.18$ & 0.0045 & $8.048 \pm 2.07$ & 0.0088 & 0.1064 \\
\hline \multirow{6}{*}{$\begin{array}{l}\text { ART } \\
\text { Max }\end{array}$} & Preoperative & $175.021 \pm 67.32$ & & $169.881 \pm 55.36$ & & 0.594 \\
\hline & 1 year & $163.892 \pm 66.99$ & 0.2599 & $152.052 \pm 48.59$ & 0.0365 & 0.1994 \\
\hline & 2 years & $161.924 \pm 66.99$ & 0.1852 & $148.644 \pm 48.68$ & 0.0131 & 0.1506 \\
\hline & 3 years & $160.451 \pm 67.09$ & 0.141 & $146.292 \pm 48.34$ & 0.0026 & 0.0763 \\
\hline & 4 years & $159.344 \pm 67.07$ & 0.1133 & $145.287 \pm 48.08$ & 0.0026 & 0.0918 \\
\hline & 5 years & $158.86 \pm 67.12$ & 0.1028 & $144.092 \pm 48.12$ & 0.0026 & 0.1093 \\
\hline
\end{tabular}

$\Delta \mathrm{K}=-1.85 \mathrm{D}$ in the accelerated group, $p=0.36)$. Several other studies [22, 29, 31, 48] showed comparable successful clinical results regarding the two procedures. Moreover, Yildirim et al. [49] had published the results of a study which compared two different types of accelerated CXL $\left(30 \mathrm{mw} / \mathrm{cm}^{2}\right.$ for 4 minutes and $18 \mathrm{~mW} / \mathrm{cm}^{2}$ for 5 minutes) and revealed no statistically significant changes in topographical and corneal measurements between the two groups. Furthermore, Mazzota et al. [50] demonstrated better functional results and deeper stromal penetration in pulsed-light accelerated CXL compared to continuous light accelerated CXL treatment with a follow-up of one year. Scherif [51] compared the Dresden protocol with the accelerated CXL $\left(30 \mathrm{~mW} / \mathrm{cm}^{2} 4\right.$ minutes 20 seconds) and demonstrated that there was no statistically significant difference between the two groups in terms of clinical results.
Conflicting studies showed that conventional CXL improved BCVA and decreased Kmax and Kmean, but the accelerated procedure provided unchanged BCVA, Kmax, and Kmean [52]. Moreover, Choi et al. [53] and Peyman et al. [54] revealed that an accelerated KCL procedure of $30 \mathrm{~mW} / \mathrm{cm}^{2} 3$ minutes 40 seconds provided a lower efficiency compared with the Dresden protocol, demonstrated by the depth of the demarcation line. It was postulated that the reduced effect of $30 \mathrm{~mW} /$ $\mathrm{cm}^{2}$ in 3-4 minutes is linked with the decrease in oxygen in these high-fluence treatments [55]. The introduction of pulse treatments aimed to restore oxygen in the cornea [55].

Our findings showed a statistically significant reduction in Kmax compared to baseline in both groups $(p<0.05)$ which changed during the 5 years of follow-up. We used this parameter not only to establish the progression of 
TABLE 4: Abberometric parameter evolution in both groups.

\begin{tabular}{|c|c|c|c|c|c|c|}
\hline & Parameter & S-CXL & $\begin{array}{l}p \text { value compared to } \\
\text { baseline }\end{array}$ & A-CXL & $\begin{array}{c}p \text { value compared to } \\
\text { baseline }\end{array}$ & $\begin{array}{c}p \text { value between } \\
\text { groups }\end{array}$ \\
\hline \multirow{6}{*}{$\mathrm{HOA}$} & Preoperative & $8.383 \pm 3.81$ & & $8.962 \pm 2.93$ & & 0.2785 \\
\hline & 1 year & $7.745 \pm 3.65$ & 0.0244 & $8.311 \pm 3.16$ & 0.018 & 0.28 \\
\hline & 2 years & $7.733 \pm 3.64$ & 0.0236 & $8.224 \pm 3.43$ & 0.0122 & 0.3617 \\
\hline & 3 years & $7.719 \pm 3.65$ & 0.0226 & $8.164 \pm 3.38$ & 0.0099 & 0.3992 \\
\hline & 4 years & $7.709 \pm 3.65$ & 0.0219 & $7.998 \pm 3.21$ & 0.0086 & 0.3881 \\
\hline & 5 years & $7.706 \pm 3.65$ & 0.0217 & $7.989 \pm 3.03$ & 0.0067 & 0.3151 \\
\hline \multirow{6}{*}{ RMS } & Preoperative & $193.823 \pm 16.63$ & & $195.142 \pm 14.1$ & & 0.584 \\
\hline & 1 year & $187.370 \pm 16.78$ & 0.0092 & $193.120 \pm 13.75$ & 0.0372 & 0.175 \\
\hline & 2 years & $186.374 \pm 16.73$ & 0.0027 & $192.820 \pm 13.52$ & 0.0305 & 0.177 \\
\hline & 3 years & $185.373 \pm 16.68$ & 0.0007 & $192.670 \pm 13.74$ & 0.0275 & 0.269 \\
\hline & 4 years & $185.186 \pm 16.71$ & 0.0005 & $192.446 \pm 13.66$ & 0.0206 & 0.22 \\
\hline & 5 years & $185.075 \pm 16.73$ & 0.0004 & $192.388 \pm 13.7$ & 0.019 & 0.118 \\
\hline
\end{tabular}

keratoconus, but also as an indicator of successful results in CXL. Similarly, Kirgiz et al. [32] reported in a comparative study between two procedures of accelerated CXL $(18 \mathrm{~mW} /$ $\mathrm{cm}^{2}$ in 5 minutes and $9 \mathrm{~mW} / \mathrm{cm}^{2}$ in 10 minutes) that Kmax is a useful index of CXL evaluation results, with a statistically significant decrease in accelerated CXL in 10 minutes.

We observed a decrease in both cylinder and spherical equivalents after CXL in our samples, with no statistically significant difference in the change between the study groups. Our results are similar with those reported by Woo et al. [22] who enrolled 76 patients in a prospective study and reported no difference between accelerated and standard CXL beyond the 3 months after surgery. Hashemian et al. [28] and Kanellopoulos [45] have also reported a comparable reduction in spherical equivalent and cylindrical error in both accelerated and conventional CXL.

Results on UCVA and BVCA reported in the literature are conflicting for these techniques, with either no change or an improvement reported following CXL. Wittig-Silva [56] and Coskunseven et al. [57] showed in their studies an increase in UCVA and BCVA $(p<0.01)$ compared to controls. $\mathrm{Ng}$ et al. [52] showed no significant difference in the change of BCVA at 12 months after CXL in both conventional and accelerated CXL groups. Only UCVA showed mild but significant improvement of 0.13 lines. In our study, UCVA increased in the same manner in the conventional group and the accelerated CXL group, as showed by the higher improvement at years 1 and 2 . However, the improvement became comparable from the first year after the procedure. Our findings revealed that BCVA in the conventional treated group had a higher improvement at 1 year compared to the accelerated one but stabilized thereafter. Thus, at 3, 4, and 5 years following the procedure, patients in the accelerated group presented an improved BCVA compared to the conventional group ( $p<0.05$ for both time points). The results in BCVA improvement in the conventional group are similar with those we published before [19].

In our study, we evaluated the HOA and RMS parameters, and we demonstrated a statistically significant reduction in both groups compared with baseline. Similarly, Greenstein et al. [58] revealed a significant decrease in HOA, total coma, three-order coma, and vertical coma. Moreover,
Caporossi et al. [14] showed a significant decline of HOA and coma aberration as early and long-term results (2 years), following CXL. Kirgiz et al. [32] demonstrated a real improvement in coma values in the accelerated CXL with 10 minutes at 1 -year follow-up.

Our findings showed a statistically significant reduction in the topographical index (TP), corneal volume, ISV, IHA, IHT, BAD_D, and ART Max from baseline, but there was no significant difference between the two groups $(p<0.05$ at all time points). Similarly, Kirgiz et al. [32] revealed the same results regarding the above parameters.

Demarcation line was limited to the anterior-mid stroma until there was a maximum depth of $202 \pm 12.03 \mu \mathrm{m}$ in the accelerated group when compared with conventional CXL that reached $214 \pm 12.82 \mu \mathrm{m}$.

Doors showed similar results [38] and described the best visibility of the corneal demarcation line using AS-OCT at 1 month after CXL treatment with an average of depth of $313 \mu \mathrm{m}$. Wollensak et al. [11] described cellular apoptosis to a depth of $300 \mu \mathrm{m}$ radiating with UVA at $3 \mathrm{~mW} / \mathrm{cm}^{2}$. Furthermore, similar results were demonstrated by Seiler and Hafezi [59], Kymionis et al. [60], Doors et al. [38], and Yam et al. [36]. In accelerated CXL, Seiler and Hafezi [59], Moramarco et al. [37], and Kymionis et al. [60] demonstrated a demarcation line depth of $213-215 \mu \mathrm{m}$ after 1 month following the procedure. Similar to our observation, Kimionis et al. [60] noted that a 10-minute treatment with $9 \mathrm{~mW} / \mathrm{cm}^{2}$ resulted in a demarcation line that was less deep $(288.46 \pm 42.37 \mu \mathrm{m})$ compared to the standard procedure $(350.75 \pm 49.34 \mu \mathrm{m})$. They also reported that a modified accelerated protocol of CXL $\left(9 \mathrm{mw} / \mathrm{cm}^{2}\right.$ for 14 minutes) provides the same demarcation line depth as the conventional procedure $(294.38 \pm 60.57 \mu \mathrm{m}$ in the accelerated group and $380.78 \pm 54.99 \mu \mathrm{m}$ in the conventional group). The difference was not statistically significant. Kymionis [60] and Mazzota et al. [61] demonstrated a deep demarcation line of $280 \mu \mathrm{m}$ in the pulsed-light cross-linking procedure applied for 6 minutes.

This paper has several limitations that should be acknowledged. This was a retrospective study, and there was no randomization. However, the strength of our paper comes from the relatively large sample size compared to previous reports and on the long-term follow-up of these 
patients, adding to previous knowledge on this subject. To our knowledge, this is the first comparative study done in Romania and is the first one with such a long period of follow-up (5 years).

\section{Conclusion}

Our study revealed the efficacy and safety of long-term follow-up (5 years) in accelerated CXL in comparison with the conventional protocol. Following both protocols, a stabilization of $\mathrm{KCN}$ after 1 year was obtained.

\section{Data Availability}

The data used and analyzed in the present study are available from the corresponding author.

\section{Disclosure}

The results were presented previously at the European Contact Lens and Ocular Surface Congress, London, UK, December 2017.

\section{Conflicts of Interest}

The authors declare that they have no conflicts of interest.

\section{Authors' Contributions}

Cristina Ariadna Nicula conceived the study and was the major contributor in surgeries, in writing the manuscript, and in collecting data. Dorin Nicula participated in surgeries and contributed in its design and coordination. Anca Rednik helped to collect the data, made the statistical analysis, and drafted the manuscript. Adriana Elena Bulboaca made the analysis and interpretation of data. All authors have read and approved the final manuscript.

\section{References}

[1] Y. S. Rabinowitz, "Keratoconus," Survey of Ophthalmology, vol. 42, no. 4, pp. 297-319, 1998.

[2] J. L. O. Jiménez, J. C. G. Jurado, F. J. B. Rodriguez, and D. S. Laborda, "Keratoconus. age of onset and natural history," Optometry and Vision Science, vol. 74, no. 3, pp. 147151, 1997.

[3] K. Miháltz, I. Kovács, Á. Takács, and Z. Z. Nagy, "Evaluation of keratometric, pachymetric, and elevation parameters of keratoconic corneas with Pentacam," Cornea, vol. 28, no. 9, pp. 976-980, 2009.

[4] U. de Sanctis, C. Loiacono, L. Richiardi, D. Turco, B. Mutani, and F. M. Grignolo, "Senzitivity and specificity of posterior corneal elevation measured by Pentacamin discriminating keratoconus/subclinical keratoconus," Ophthalmology, vol. 115, no. 9, pp. 1534-1539, 2008.

[5] G. Wollensak, "Crosslinking treatment of progressive keratoconus: new hope," Current Opinion in Ophthalmology, vol. 17, no. 4, pp. 356-360, 2006.

[6] E. Spoerl, A. Hoyer, L. E. Pillunat, and F. Raiskup, "Corneal cross-linking and safety issues," The Open Ophthalmology Journal, vol. 5, no. 1, pp. 14-16, 2011.
[7] E. Spoerl, M. Huhle, and T. Seiler, "Induction of cross-links in corneal tissue," Experimental Eye Research, vol. 66, no. 1, pp. 97-103, 1998.

[8] Y. Zhang, A. A. Broekhuis, M. C. A Stuart et al., "Crosslinking of multiwalled carbon nanotubes with polymeric amines," Macromolecules, vol. 41, no. 16, pp. 6141-6146, 2008.

[9] G. Wollensak, E. Spoerl, and T. Seiler, "Riboflavin/ultravioleta-induced collagen crosslinking for the treatment of keratoconus," American Journal of Ophthalmology, vol. 135, no. 5, pp. 620-627, 2003.

[10] K. Lu, W. Ye, L Zhou et al., "Structural characterization of formaldehyde-induced cross-links between amino acids and deoxynucleosides and their oligomers," Journal of the American Chemical Society, vol. 132, no. 10, pp. 3388-3399, 2010.

[11] G. Wollensak, E. Spoerl, M. Wilsch, and T. Seiler, "Keratocyte apoptosis after corneal collagen cross-linking using riboflavin/UVA treatment," Cornea, vol. 23, no. 1, pp. 43-49, 2004.

[12] E. Chan and G. R. Snibson, "Current status of corneal collagen cross-linking for keratoconus:a review," Clinical and Experimental Optometry, vol. 96, no. 2, pp. 155-164, 2013.

[13] F. Raiskup-Wolf, A. Hoyer, E. Spoerl, and L. E. Pillunat, "Collagen crosslinking with riboflavin and ultraviolet-A light in keratoconus: long term results," Journal of Cataract \& Refractive Surgery, vol. 34, no. 5, pp. 796-801, 2008.

[14] A. Caporossi, C. Mazzotta, S. Baiocchi, and T. Caporossi, "Long term results of riboflavin ultraviolet a corneal collagen cross-linking for keratoconus in Italy: the Siena eye cross study," American Journal of Ophthalmology, vol. 149, no. 4, pp. 585-593, 2010.

[15] C. Wittig-Silva, M. Whiting, E. Lamoureux, R.G. Lindsday, L. J. Sullivan, and G. R. Snibson, "A randomised controlled trial of corneal collagen cross-linking in progressive keratoconus: preliminary results," Journal of Refractive Surgery, vol. 24, no. 7, pp. S720-S725, 2008.

[16] A. Caporossi, C. Mazzotta, S. Baiocchi, T. Caporossi, and R. Denaro, "Age related long-term functional results after riboflavin UV A corneal cross-linking," Journal of Ophthalmology, vol. 2011, pp. 1-6, 2011.

[17] A. Caporossi, C. Mazzotta, S. Baiocchi, T. Caporossi, R. Denaro, and A. Balestrazzi, "Riboflavin-UVA-induced corneal collagen cross-linking in pediatric patients," Cornea, vol. 31, no. 3, pp. 227-231, 2012.

[18] C. Nicula, D. Nicula, and R. N Pop, "Results at 7 years after cross-linking procedure in keratoconic patients," Journal Français d'Ophthalmology, vol. 40, no. 7, pp. 535-541, 2017.

[19] C. Nicula, R. Pop, A. Rednik, and D. Nicula, "10-year results of standard cross-linking in patients with progressive keratoconus in Romania," Journal of Ophthalmology, vol. 2019, pp. 1-5, 2019.

[20] S. Schumacher, L. Oeftiger, and M. Mrochen, "Equivalence of biomechanical changes induced by rapid and standard corneal cross-linking, using riboflavin and ultraviolet radiation," Investigative Ophthalmology \& Visual Science, vol. 52, no. 12, pp. 9048-9052, 2011.

[21] A. Hammer, O. Richoz, S. Arba Moswuera, D. Tabibian, F. Hoogewoud, and F. Hafezi, "Corneal biomechanical properties at different corneal cross-linking (CXL) irradiances," Investigative Opthalmology \& Visual Science, vol. 55, no. 5, pp. 2881-2884, 2014.

[22] J. H. Woo, J. V. Iyer, L Lim et al., "Conventional versus accelerated collagen cross-linking for keratoconus: a comparison of visual, refractive, topographic and biomechanical outcomes," The Open Ophthalmology Journal, vol. 11, no. 1, pp. 262-272, 2017. 
[23] A. B. Cummings, R. McQuaid, S. Naughton, E. Brennan, and M. Mrochen, "Optimizing corneal cross-linking in the treatment of keratoconus:a comparison of outcomes after standard and high -intensity protocols," Cornea, vol. 35, no. 6, pp. 814-822, 2016.

[24] D. Touboul, N. Efron, D. Smadja, D. Praud, F. Malet, and J. Colin, "Corneal confocal microscopy following conventional, transepithelial and accelerated corneal collagen crosslinking proceduresfor keratoconus," Journal of Refractive Surgery, vol. 28, no. 11, pp. 769-776, 2012.

[25] M. Shajari, G. Sowwa, G. Steinwender et al., "Comparison of corneal collagen cross-linking protocolsmeasured with Scheimpflug tomography," Cornea, vol. 37, no. 7, pp. 870874, 2018.

[26] H. Hashemi, R. Ambrósio, R. Vinciguerra et al., "Two-year changes in corneal stiffness parameters after accelerated corneal cross-linking," Journal of Biomechanics, vol. 93, pp. 209-212, 2019.

[27] N. H. Aldahlawi, S. Hayes, D. P. S. O’Brart, and K. M. Meek, "Standard versus accelerated riboflavin-ultraviolet corneal collagen crosslinking: resistance against enzymatic digestion," Journal of Cataract \& Refractive Surgery, vol. 41, no. 9, pp. 1989-1996, 2015.

[28] H. Hashemi, A. Fotouhi, M. Miraftab et al., "Short-term comparison of accelerated and standard methods of corneal collagen crosslinking," Journal of Cataract \& Refractive Surgery, vol. 41, no. 3, pp. 533-540, 2015.

[29] U. Elbaz, C. Shen, A Lichtinger et al., "Accelerated versus standard corneal collagen crosslinking combined with same day phototherapeutic keratectomy and single intrastromal ring segment implantation forkeratoconus," British Journal of Ophthalmology, vol. 99, no. 2, pp. 155-159, 2015.

[30] M. Tomita, M. Mita, and T. Huseynova, "Accelerated versus conventional corneal collagen crosslinking," Journal of Cataract \& Refractive Surgery, vol. 40, no. 6, pp. 1013-1020, 2014.

[31] M. M. Sadoughi, B. Einollahi, A. Baradaran-Rafii, D. Roshandel, H. Hasani, and M. Nazeri, "Accelerated versus conventional corneal collagen cross-linking in patients with keratoconus: an intrapatient comparative study," International Ophthalmology, vol. 38, no. 1, pp. 67-74, 2016.

[32] A. Kirgiz, M. Eliack, and Y. Yildirim, "Different accelerated corneal collagen cross-linking treatment modalities in progressive keratoconus," Eye and Vision, vol. 16, no. 6, pp. 1-32, 2019.

[33] E. Vounotrypidis, A. Athanasiou, K. Kortum et al., "Longterm data base analysis of conventional and accelerated crosslinked keratoconic mid-European eyes," Graefe's Archive for Clinical and Experimental Ophthalmology, vol. 256, no. 6, pp. 1165-1172, 2018.

[34] M. Rechichi, C. Mazzotta, S. Daya, R. Mencucci, M. Lanza, and A. Meduri, "Intraoperative OCT pachymetry in patients undergoing dextran-free riboflavin UVA accelerated corneal collagen crosslinking," Current Eye Research, vol. 41, no. 10, pp. 1310-1315, 2016.

[35] L. Spadea, E. Tonti, and E. Vingolo, "Corneal stromal demarcation line after collagen cross-linking in corneal ectatic diseases:a review of the literature," Clinical Ophthalmology, vol. 10, pp. 1803-1810, 2016.

[36] J. C. S Yam, C. W. N Chan, and A. C. K Cheng, "Corneal collagen cross-linking demarcation line depth assessed by Visante OCT after CXL for keratoconus and corneal ectasia," The Journal of Refractive Surgery, vol. 28, no. 7, pp. 475-481, 2012.
[37] A. Moramarco, A. Iovieno, A. Sartori, and L. Fontana, "Corneal stromal demarcation line afteraccelerated crosslinking using continuous and pulsed light," Journal of Cataract \& Refractive Surgery, vol. 41, no. 11, pp. 2546-2551, 2015.

[38] M. Doors, N. G. Tahzib, F. A. Eggink, T. T. J. M Berendschot, C. A. B Webers, and R. M. M. A Nujits, "Use of anterior segment optical coherence tomography to study corneal changes after collagen cross-linking," American Journal of Ophthalmology, vol. 148, no. 6, pp. 844-851, 2009.

[39] P. Vinciguerra, E. Albe, S. Trazza et al., "Refractive, topographic, tomographic, and aberrometric analysis of keratoconic eyes undergoing corneal cross-linking," Ophthalmology, vol. 116, no. 3, pp. 369-378, 2009.

[40] M. A. Henriquez, S. Villegas, M. Rincon, C. Maldano, and L. Izquierdo, "Long-term efficacy and safety after corneal collagen crosslinking in pediatric patients: three-year followup," The European Journal of Ophthalmology, vol. 28, no. 4, pp. $415-418,2018$.

[41] V. Galvis, A. Tello, and A. I. Ortiz, "Corneal collagen crosslinking with riboflavin and ultraviolet forkeratoconus: long-term follow-up," Journal of Cataract \& Refractive Surgery, vol. 41, no. 6, pp. 1336-1337, 2015.

[42] M. De Bernardo, L. Capasso, M. Lanza et al., "Long-termresults of corneal collagen crosslinking for progressive keratoconus," Journal of Optometry, vol. 8, no. 3, pp. 180-186, 2015.

[43] F. Raiskup, A. Theuring, L. E. Pillunat, and E. Spoerl, "Corneal collagen crosslinking with riboflavinand ultraviolet-A light in progressive keratoconus: ten-year results," Journal of Cataract \& Refractive Surgery, vol. 41, no. 1, pp. 41-46, 2015.

[44] T. Koller, H. Iseli, F. Hafezi, P. Vinciguerra, and T. Seiler, "Scheimflug imaging of corneas after collagen cross-linking," Cornea, vol. 28, no. 5, pp. 510-515, 2009.

[45] J. Kanellopoulos, "Long term results of a prospective randomized bilateral eye comparison trial of higher fluence, shorter duration ultraviolet A radiation, and riboflavin collagen crosslinking for progressive keratoconus," Clinical Ophthalmology, vol. 6, pp. 97-101, 2012.

[46] R. Shetty, N. K. Pahuja, R. M. M. A Nuijts et al., "Current protocols of corneal collagen cross-linking:visual, refractive, and tomographic outcomes," American Journal of Ophthalmology, vol. 160, no. 2, pp. 243-249, 2015.

[47] H. Hashemian, M. Jabbarvand, M. Khodaparast, and K. Ameli, "Evaluation of corneal changes after conventional versus accelerated corneal cross-linking: a randomized controlled trial," Journal of Refractive Surgery, vol. 30, no. 12, pp. 837-842, 2014.

[48] K. U. Kortuem, E. Vounotrypidis, A Athanasiou et al., "Differences in corneal clinical findings after standard and accelerated cross-linking in patients with progressive keratoconus," BMC Ophthalmology, vol. 17, no. 1, p. 222, 2017.

[49] Y. Yildirim, O. Olcucu, Z. K. Gunaydin et al., "Comparison of accelerated corneal collagen cross-linking types for treating keratoconus," Current Eye Research, vol. 42, no. 7, pp. 971-975, 2017.

[50] C. Mazzotta, C. Traversi, S. Caragiuli, and M. Rechichi, "Pulsed vs continuous light accelerated corneal collagen crosslinking: in vivo qualitative investigation by confocal microscopy and corneal OCT," Eye, vol. 28, no. 10, pp. 1179-1183, 2014.

[51] A. M. Sherif, "Accelerated versus conventional corneal collagen cross-linking in the treatment of mild keratoconus: a 
comparative study," Clinical Ophthalmology, vol. 8, pp. 1435-1440, 2014.

[52] A. L. Ng, T. C. Chan, and A. C. Cheng, "Conventional versus accelerated corneal collagen cross-linkingin the treatment of keratoconus," Clinical \& Experimental Ophthalmology, vol. 44, no. 1, pp. 8-14, 2016.

[53] M. Choi, J. Kim, E. K. Kim, K. Y. Seo, and T.-i. Kim, "Comparison of the conventional Dresden protocol and accelerated protocol with higher ultraviolet intensity in corneal collagencross-linking for keratoconus," Cornea, vol. 36, no. 5, pp. 523-529, 2017.

[54] A. Peyman, A. Nouralishahi, F. Hafezi, S. Kling, and M. Peyman, "Stromal demarcation line in pulsed versus continuous light accelerated corneal cross-linking for keratoconus," Journal of Refractive Surgery, vol. 32, no. 3, pp. 206-208, 2016.

[55] L. Lim and E. W. L. Lim, "A review of corneal collagen crosslinking - current trends in practice applications," The Open Ophthalmology Journal, vol. 12, no. 1, pp. 181-213, 2018.

[56] C. Wittig-Silva, E. Chan, F. M. A. Islam, T. Wu, M. Whiting, and G. R. Snibson, "A randomized, controlled trial of corneal collagen cross-linking in progressive keratoconus: three-year results," Ophthalmology, vol. 121, no. 4, pp. 812-821, 2014.

[57] E. Coskunseven, M. R. Jankov, and F. Hafezi, "Contralateral eye study of corneal collagencross-linking with riboflavin and UVA irradiation in patients with keratoconus," Journal of Refractive Surgery, vol. 25, no. 4, pp. 371-376, 2009.

[58] S. A. Greenstein, K. L. Fry, M. J. Hersch, and P. S. Hersch, "Higher-order aberrations after corneal collagen crosslinking for keratoconus and corneal ectasia," Journal of Cataract \& Refractive Surgery, vol. 38, no. 2, pp. 292-302, 2012.

[59] T. Seiler and F. Hafezi, "Corneal cross-linking induced stromal demarcation line," Cornea, vol. 25, no. 9, pp. 10571059, 2006.

[60] G. D. Kymionis, K. I. Tsoulnaras, M. A. Grentzelos et al., "Corneal stromal demarcation line after standard and high intensity collagen cross-linking determined with anterior segment optical coherence tomography," Journal of Cataract \& Refractive Surgery, vol. 40, no. 5, pp. 736-740, 2014.

[61] C. Mazzotta, S. Baiocchi, S. A. Bagaglia, M. Fruschelli, A. Meduri, and M. Rechichi, "Accelerated $15 \mathrm{~mW}$ pulsed-light crosslinking to treat progressive keratoconus: two-year clinical results," Journal of Cataract \& Refractive Surgery, vol. 43, no. 8, pp. 1081-1088, 2017. 